\title{
Effect of Caregiver Driven Robot-Assisted In-Ward Training in Subacute Stroke Patients: A Case Series
}

\author{
Sang Beom Kim, MD, Kyeong Woo Lee, MD, Jong Hwa Lee, MD, \\ Sook Joung Lee, MD, Jin Gee Park, MD, Joo Won Park, MD
}

Department of Physical Medicine and Rehabilitation and Regional Cardiocerebrovascular Center, Dong-A University College of Medicine, Busan, Korea

Objective To evaluate the effect of caregiver driven robot-assisted in-ward training in subacute stroke patients. Methods A retrospective evaluation was performed for patients treated with caregiver driven robot-assisted inward training to retain gait function from June 2014 and December 2016. All patients received more than 2 weeks of caregiver driven robot-assisted in-ward training after undergoing conventional programs. The robot was used as a sitting device, a standing frame, or a high-walker depending on functional status of the patient. Patients were evaluated before and after robot training. Patient records were assessed by Korean version of Modified Barthel Index (K-MBI), Functional Independence Measure (FIM), and Functional Ambulation Category (FAC).

Results Initially, patients used the robot as a sitting device $(n=6)$, a standing frame $(n=7)$, or a partial body-weight support high-walker $(\mathrm{n}=2)$. As patient functions were improved, usage level of the robot was changed to the next level. At the end of the treatment, the robot was used as a sitting device $(n=1)$, a standing frame $(n=6)$, or highwalker $(\mathrm{n}=8)$. Scores of K-MBI $(\Delta 17.47 \pm 10.72)$ and FIM $(\Delta 19.80 \pm 12.34)$ were improved in all patients.

Conclusion Patients' usage level of the robot and functional scores were improved. Therefore, performing additional caregiver driven robot-assisted in-ward training is feasible and beneficial for subacute stroke patients.

Keywords Stroke rehabilitation, Stroke, Robot

\section{INTRODUCTION}

The purpose of stroke rehabilitation is to improve func- tion, prevent deterioration of function, and achieve the highest possible level of independence within limits presented by persistent stroke impairments [1]. From acute

Received June 2, 2017; Accepted July 26, 2017

Corresponding author: Joo Won Park

Department of Physical Medicine and Rehabilitation, Dong-A Medical Center, Dong-A University College of Medicine, 26 Daesingongwon-ro, Seo-gu, Busan 49201, Korea. Tel: +82-51-240-5691, Fax: +82-51-254-8511, E-mail: exception7@naver.com

ORCID: Sang Beom Kim (http://orcid.org/0000-0002-5622-5933); Kyeong Woo Lee (http://orcid.org/0000-0001-8785-5535); Jong Hwa Lee (http:// orcid.org/0000-0003-2489-358X); Sook Joung Lee (http://orcid.org/0000-0002-6894-445X); Jin Gee Park (http://orcid.org/0000-0003-2672-1618); Joo Won Park (http://orcid.org/0000-0003-4969-3824).

(c) This is an open-access article distributed under the terms of the Creative Commons Attribution Non-Commercial License (http://creativecommons.org/ licenses/by-nc/4.0) which permits unrestricted noncommercial use, distribution, and reproduction in any medium, provided the original work is properly cited. Copyright ( 2018 by Korean Academy of Rehabilitation Medicine 
to subacute stages, there are strong evidences that increased therapeutic activity can lead to better post-stroke outcome [2-5]. Recently, an intensive rehabilitation program ( 3 hours per day for 5 days per week) has been recommended for a subacute stroke phase inpatient rehabilitation facility [6]. However, hospitalized patients in Korea who need extensive rehabilitation treatment spend more than $50 \%$ of the daytime resting alone in their hospital rooms. These findings indicate that patients need additional considerable time to engage in therapeutic activity outside the conventional physical therapy room. Patients could achieve better function if they perform more intensive rehabilitation treatments during the subacute phase. During this period, patients with better functional status may exercise by themselves. However, those with severe symptoms such as ataxia or weakness cannot exercise on their own. For severe patients, one way to increase their exercise time is to actively involve family members (or their private caregivers) in the rehabilitation process $[7,8]$.

A recent pilot study has indicated that gait training using robots for subacute non-ambulatory patients without therapists is a safe, effective, and feasible treatment [9]. Additionally, a recent Cochrane review has revealed that caregiver-mediated exercises (CME) can improve functional ability and health-related quality of life of people with stroke [7]. These results demonstrate that CME can augment current therapeutic options for stroke rehabilitation. Stroke patients want to be able to exercise in the ward besides rehabilitation therapy at the physical therapy room. However, for severely impaired stroke patients, performing exercise alone in their ward presents restrictions due to safety concerns such as falls. To address these safety problems, robot has been used for patients who have difficulty in performing exercises by themselves in the ward.

The objective of this study was to determine the effect of caregiver driven robot-assisted in-ward training in additional to conventional rehabilitation program on balance and weakness problems in subacute stroke patients. A comparative analysis of clinical index was performed using retrospective data for 15 cases of stroke patients who received caregiver driven robot-assisted in-ward training in subacute stroke phase.

\section{MATERIALS AND METHODS}

\section{Purpose of robot use}

Four Robin-T (TES Co., Osan, Korea) units were used at Dong-A University Hospital, in Busan, Korea since February 2014. Originally, the robot was developed for transferring patients who had difficulty to move so that their activities of daily living (ADL) could be improved. However, it could not be used because of a long preparation time to put on safety equipment and its slow speed of moving. However, the safety equipment for preventing falls provided good stability. This feature could allow the robot to be used as a sitting device, a standing frame, or
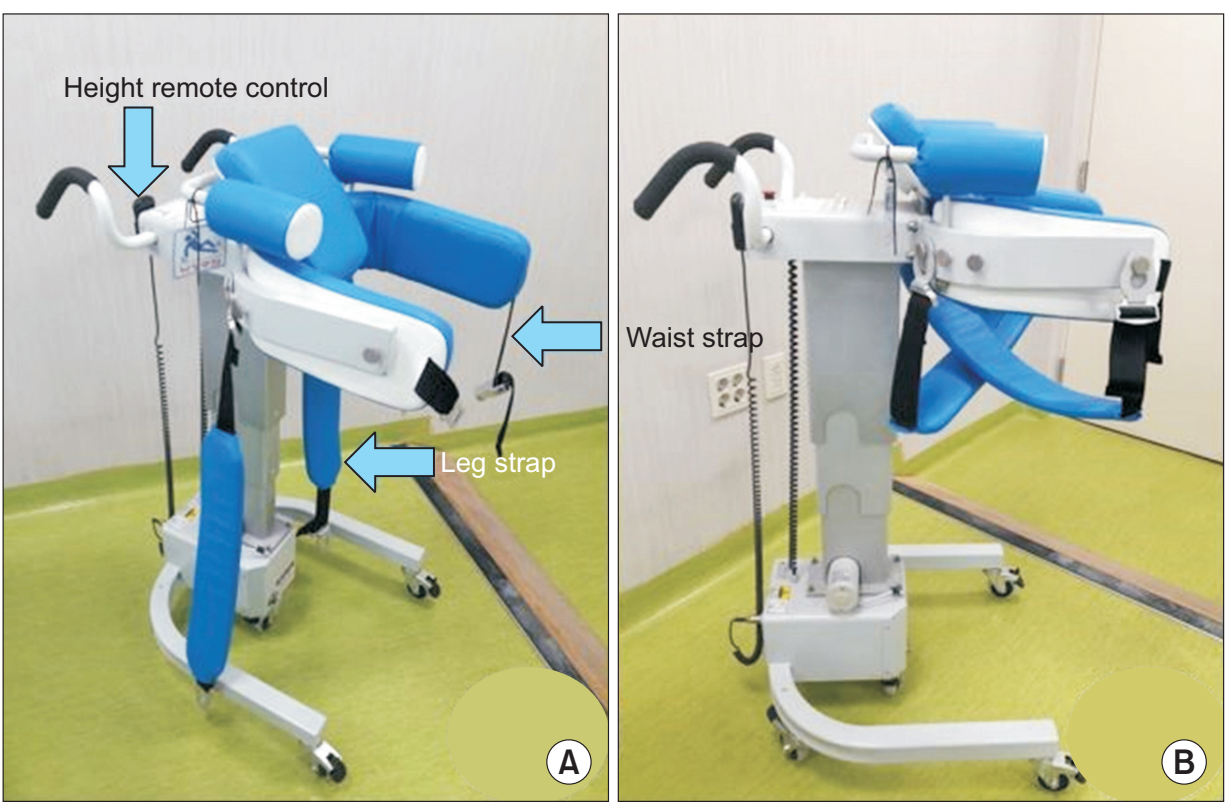

Fig. 1. Transfer assistance robot, an inward gait training device, consisted of waist and leg straps, height remote control, and handle. (A) All straps are unfastened. (B) All straps are fastened. 
a partial body-weight support high-walker. It could be utilized safely within a ward. Such robot can alert patients who want to exercise by themselves in their ward but could not walk independently due to poor balance or hemiplegia after stroke. Therefore, they could have more time for rehabilitation.

\section{Appearance of robot}

The robot weights approximately $120 \mathrm{~kg}$. It has four wheels to make it easier to move. Two straps affix the lower limbs and one strap affixes the waist. Its main frame has a handle that can be grabbed easily (Fig. 1). The two straps that affix the lower limbs become an X shape when patient wears them. It could help partial weight-bearing. The waist strap could prevent backward falling tendency. These straps combined with its heavy weight frame can protect patient from falling backwards or sideways. To get on this robot, the patient needs to sit on the bed. The caregiver places the robot in front of the patient. The patient then leans on the trunk of the robot and grabs the handle with their hands. The caregiver then helps the patient put on the waist and lower limbs straps. The height can be adjusted using a remote control attached to the robot.

\section{Therapeutic application}

According to functional status of the patient, usage level of the robot was set before treatment. Patients who were unable to maintain independent sitting balance could use the robot as a sitting device. Those who were unable to maintain independent standing balance actively could use the robot as a standing frame to improve their antigravity muscle strength, control head and trunk posture, maintain standing ability, and prepare for gait training. Patients who were unable to walk without assistance could use the robot as a high-walker to improve gait mobility and balance. When patient reached desired function during treatment, the usage level of the robot could be changed to the next level. In rehabilitation wards, the robot was used as a sitting device for patients who needed sitting balance training (Fig. 2A). Fixed wheels acted as a safe standing frame for patients who needed standing balance training and lower extremity strengthening (Fig. 2B). For patients who performed gait training with a partial body weight support device in a rehabilitation room and those who conducted gait training using a walker or cane, this robot could be used as a partial body-weight support high-walker (Fig. 2C). Using the robot, patients could ambulate around the ward and go to restroom under their caregiver's supervision or with minimal assistance. To utilize these robots in wards,
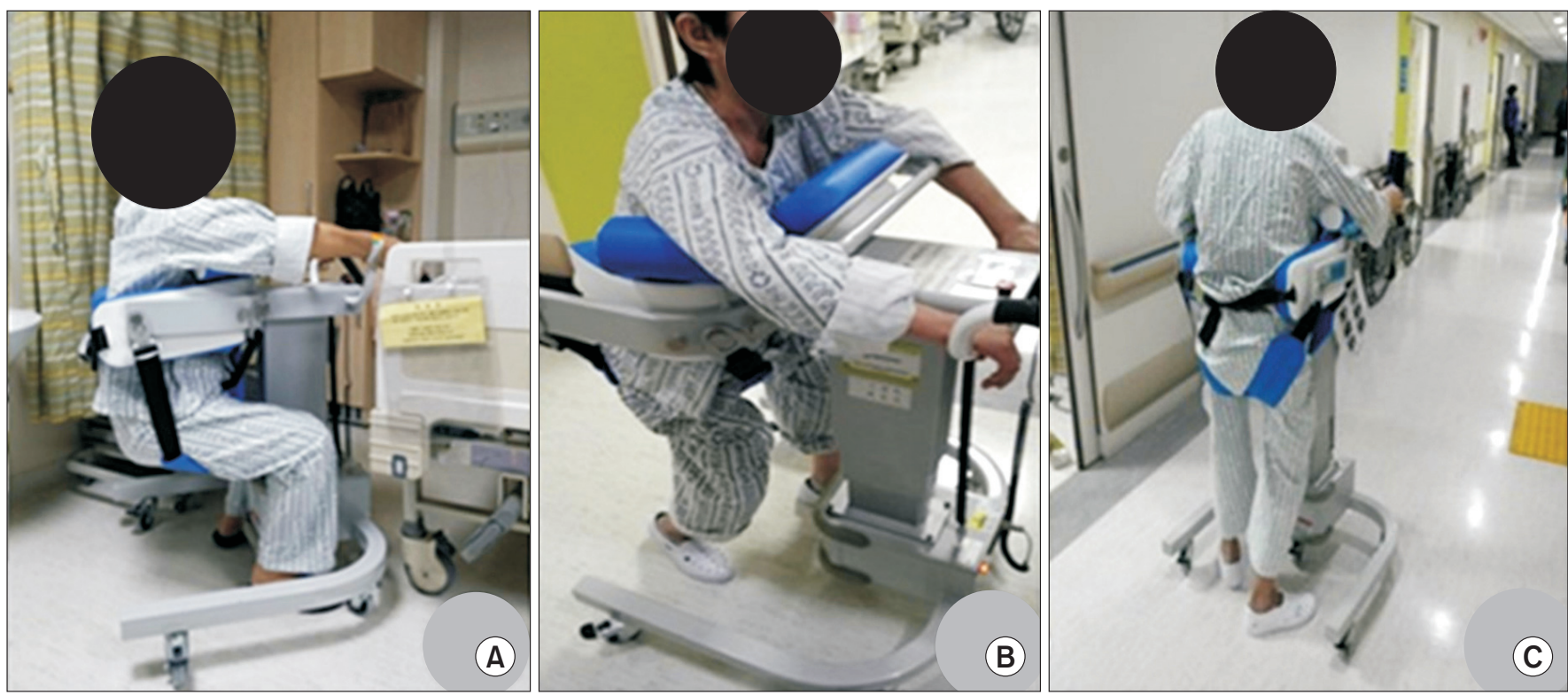

Fig. 2. Patients used body weight support device to exercise in the ward by themselves. (A) Patient could maintain sitting balance by using device in the ward. (B) Patient could maintain standing balance by using device in the ward. (C) Patient could do gait training independently by using the device in the ward. 
physicians explained to patients and caregivers on how to use the device and safety equipment. After the demonstration, patients and caregivers verified that they could understand and use the device safely as a measure of exercise by themselves. All patients' caregivers used this device independently without any help from the medical team. Patients watched television while using the device in a sitting position. It enabled safe sitting balance training and core muscle strengthening exercises.

\section{Participants and study design}

Medical records of patients who had used Robin-T were analyzed retrospectively. Patients who had been diagnosed with subacute stroke were enrolled. Subjects were referred to the Department of Physical Medicine and Rehabilitation, Dong-A University Hospital between June 2014 and December 2016 to receive rehabilitation treatment. During this period, 22 patients used these devices. Each patient used the robot at least 30 minutes per day. Among these patients, those with recurrence of stroke, cognitive impairment, or previous history of musculoskeletal issues resulting in gait problems were excluded. Patients whose caregiver were changed twice or more, those who did not comply with the guidance, and those who could not utilize the robot longer than 30 minutes per day were excluded. Retrospective review was performed for the remaining 15 patients. All patients had hemiplegia or ataxia within 3 months of stroke onset. All patients received conventional rehabilitation programs, including medication, physical and occupational therapy, and neuromuscular electrical stimulation.

Inclusion criteria were: (1) those who understood Korean, (2) those who were still in the subacute rehabilitation phase (1 week to 3 months post stroke), (3) those who had a caregiver willing to participate in the program, (4) those who could follow instructions, (5) those who had mobility problems caused by stroke (Functional Ambulation Category [FAC] score $<4$ ), and (6) those who had adequate cognition to participate (Mini-Mental State Examination score $>23$ ). Caregivers (partner, family member, or private caregiver) were eligible to participate if they were physically able to perform the exercise with the patient, understood Korean, and agreed to provide support to the patient without significant symptoms of depression.

\section{Evaluations}

All data were collected from medical records after treatment. Clinical improvement was assessed by changes between before and after caregiver driven robot-assisted in-ward training using the Korean version of Modified Barthel Index (K-MBI), Functional Independence Measure (FIM), and FAC. The K-MBI measures independence in ADL, with a maximum score of 100 . It assesses 10 ADLs: bowel control, bladder control, personal hygiene, toilet transfer, bathtub transfer, feeding, dressing, wheelchair transfer to and from bed, walking (or wheelchair management if the patient is non-ambulatory), and ascending and descending stairs. The FIM has a total of 18 items. It is also used to assess performance during tasks that can be broadly categorized as ADL, mobility, and cognition. Each item is scored from 1 to 7 , with 7 signifying complete independence or normal functioning and 1 signifying complete dependence or requiring total assistance. The total maximum score of FIM is 126 which indicates total independence. Its minimum score is 18 , indicating that full assistance is required for all 18 items. The study protocol was approved by the Institutional Review Board of Dong-A University Hospital (No. DAUHIRB-17-129).

\section{Statistical analysis}

For statistical analysis, Wilcoxon rank sum test was used. Data were analyzed using SPSS version 22.0 for Windows (IBM, Armonk, NY, USA).

\section{RESULTS}

Table 1 shows participants' characteristics at baseline. Fifteen patients (8 men and 7 women) were included in this study. The average age of these participants was 56.7 years and the average Mini-Mental State Examination score was 25.2. Six patients received sitting balance training at the physical therapy room. Seven patients received standing balance training and two patients received gait training. Five caregivers were family members of patients. The remaining 10 caregivers were those who had been employed. All patients utilized the robot for 2 to 6 weeks. Initially, patients used the robot as a sitting device $(n=6)$, a standing frame $(\mathrm{n}=7)$, or a partial body-weight support high-walker $(n=2)$. When patient function was improved, the usage level of the robot was changed to the next level. 
Table 1. Demographics and clinical characteristics of the study population $(n=15)$

\begin{tabular}{cclllccll}
\hline $\begin{array}{c}\text { Patient } \\
\text { no. }\end{array}$ & Age & Gender & $\begin{array}{c}\text { Stroke } \\
\text { type }\end{array}$ & Lesion site & $\begin{array}{c}\text { Days } \\
\text { from } \\
\text { onset }\end{array}$ & $\begin{array}{c}\text { Duration of } \\
\text { treatment } \\
\text { (wk) }\end{array}$ & $\begin{array}{c}\text { Purpose of } \\
\text { device }\end{array}$ & Caregiver \\
\hline 1 & 61 & Female & SAH & A-com & 53 & 3 & Sitting device & Daughter \\
\hline 2 & 48 & Male & ICH & Both pons & 65 & 3 & Sitting device & Wife \\
\hline 3 & 65 & Male & Infarction & Rt. ACA & 25 & 3 & Sitting device & Private caregiver \\
\hline 4 & 38 & Male & Infarction & Lt. lateral medullar & 28 & 3 & Sitting device & Private caregiver \\
\hline 5 & 60 & Female & Infarction & Rt. pons & 41 & 3 & Sitting device & Husband \\
\hline 6 & 83 & Female & Infarction & Rt. cerebellar & 24 & 3 & Sitting device & Private caregiver \\
\hline 7 & 54 & Male & Infarction & Rt. internal capsule & 18 & 3 & Sitting device & Private caregiver \\
\hline 8 & 32 & Male & ICH & Rt. basal ganglia & 14 & 3 & Standing frame & Wife \\
\hline 9 & 39 & Male & ICH & Rt. pons & 22 & 3 & Standing frame & Private caregiver \\
\hline 10 & 73 & Female & Infarction & Rt. MCA & 35 & 6 & Standing frame & Private caregiver \\
\hline 11 & 35 & Female & ICH & Both pons & 61 & 3 & Standing frame & Parents \\
\hline 12 & 72 & Female & SAH & A-com & 55 & 3 & Standing frame & Private caregiver \\
\hline 13 & 66 & Male & Infarction & Lt. lateral medullar & 48 & 3 & Standing frame & Private caregiver \\
\hline 14 & 60 & Female & ICH & Rt. basal ganglia & 27 & 2 & PBWSHW & Private caregiver \\
\hline 15 & 65 & Male & Infarction & Lt. cerebellar & 58 & 3 & PBWSHW & Private caregiver \\
\hline
\end{tabular}

SAH, subarachnoid hemorrhage; ICH, intracerebral hemorrhage; A-com, anterior communicating artery; MCA, middle cerebral artery; Rt., right; Lt., left; PBWSHW, partial body-weight support high-walker.

At the time of treatment termination, only one patient used the robot as a sitting device. Six patients used it as a standing frame while eight patients used it as a partial body-weight support high-walker. Scores of K-MBI $(\Delta 17.47 \pm 10.72)$ and FIM $(\Delta 19.80 \pm 12.34)$ were improved in all patients. The score of FAC $(\Delta 1.07 \pm 0.46)$ was improved in fourteen patients (Table 2). There were no reported side effects such as falls or pressure ulcer from the strap.

\section{Patient case using the robot as a sitting device}

In the present study, Case \#2 (bilateral pontine hemorrhage, a 48-year-old man) was transferred to the rehabilitation center at 65 days after the onset of stroke. The patient exhibited dysarthria, dysphagia, and mild double hemiplegia and severe ataxia on both upper and lower limbs, all of which greatly inhibited ADL. He initially presented with severe ataxia and weakness on both sides. He was poor at weight bearing and balance. Thus, maximal assistance by the physical therapist was needed for training. In the ward, sitting balance training at bedside presented limitations. He could not maintain a sitting posture without maximal support. We used the device to assist him with sitting balance training. With the X-figure leg strap and waist support strap, the patient could make a chair sitting posture (Fig. 2A). Using this robot for sitting balance training, the patient's erector muscles of the spine seemed to be getting stronger. He could maintain sitting balance independently without any back support. He was encouraged to get out of the bed during the day. At discharge, he could stand up with moderate assistance.

\section{Patient case using the robot as a standing frame}

The most dramatic improvement in K-MBI and FIM scores was observed in Case \#5 (right pontine infarction, a 60-year-old woman). She was transferred to the rehabilitation center at 41 days after the onset of stroke. The patient exhibited left hemiplegia and ataxia on the upper and lower left limbs. She had difficulty of gait initiation with backward falling tendency. Thus, supervision or minimal assistance by a physical therapist was needed for standing balance training. In the ward, static standing balance training by a caregiver was dangerous due to her poor balance. To support her body weight, the robot was used. With waist support strap and handle, the patient demonstrated increased stability. She could perform inward standing training under a caregiver's supervision (Fig. 2B). Using this device for therapeutic exercise was 
Sang Beom Kim, et al.

Table 2. Changes in K-MBI, FIM, and purpose of device between pre- and post-treatment

\begin{tabular}{|c|c|c|c|c|c|c|c|c|c|c|c|}
\hline \multirow{2}{*}{$\begin{array}{c}\text { Patient } \\
\text { no. }\end{array}$} & \multicolumn{3}{|c|}{ K-MBI } & \multicolumn{3}{|c|}{ FIM } & \multicolumn{3}{|c|}{ FAC } & \multicolumn{2}{|c|}{ Purpose of device } \\
\hline & Pre & Post & $\Delta$ & Pre & Post & $\Delta$ & Pre & Post & $\Delta$ & Pre & Post \\
\hline 1 & 18 & 42 & 26 & 39 & 64 & 25 & 0 & 1 & 1 & Sitting device & Standing frame \\
\hline 2 & 10 & 32 & 22 & 28 & 55 & 27 & 0 & 1 & 1 & Sitting device & Standing frame \\
\hline 3 & 21 & 37 & 16 & 42 & 58 & 16 & 0 & 1 & 1 & Sitting device & Standing frame \\
\hline 4 & 25 & 31 & 6 & 46 & 55 & 9 & 0 & 1 & 1 & Sitting device & Standing frame \\
\hline 5 & 14 & 65 & 41 & 32 & 88 & 56 & 0 & 2 & 2 & Sitting device & PBWSHW \\
\hline 6 & 15 & 25 & 10 & 33 & 45 & 12 & 0 & 1 & 1 & Sitting device & Standing frame \\
\hline 7 & 29 & 53 & 24 & 50 & 75 & 25 & 1 & 2 & 1 & Standing frame & Standing frame \\
\hline 8 & 49 & 55 & 6 & 70 & 78 & 8 & 2 & 3 & 1 & Standing frame & PBWSHW \\
\hline 9 & 26 & 67 & 31 & 46 & 85 & 29 & 1 & 3 & 2 & Standing frame & PBWSHW \\
\hline 10 & 29 & 56 & 27 & 49 & 72 & 23 & 1 & 2 & 1 & Standing frame & PBWSHW \\
\hline 11 & 75 & 83 & 8 & 98 & 108 & 10 & 2 & 3 & 1 & Standing frame & PBWSHW \\
\hline 12 & 37 & 54 & 17 & 58 & 78 & 20 & 1 & 2 & 1 & Standing frame & PBWSHW \\
\hline 13 & 38 & 44 & 6 & 58 & 67 & 9 & 1 & 2 & 1 & Standing frame & $\begin{array}{l}\text { Standing balance } \\
\text { training }\end{array}$ \\
\hline 14 & 58 & 66 & 8 & 78 & 90 & 12 & 3 & 3 & 0 & PBWSHW & PBWSHW \\
\hline 15 & 52 & 66 & 14 & 72 & 88 & 16 & 2 & 3 & 1 & PBWSHW & PBWSHW \\
\hline
\end{tabular}

K-MBI, Korean version of Modified Barthel Index; FIM, Functional Independent Measure; FAC, Functional Ambulation Categories; PBWSHW, partial body-weight support high-walker.

effective in improving gait and balance function and ADL $(p<0.05)$ for this patient. She and the caregiver (her husband) could independently use the device without help from the medical team. At discharge, she could walk through support from a walker with moderate assistance.

\section{Patient case using robot as a partial body-weight support high-walker}

Case \#15 (left cerebellar infarction, a 65-year-old man) was transferred to the rehabilitation center at 58 days after the onset of stroke. Although his muscle powers on the left upper and lower limbs were relatively well maintained, appropriate motor functions could not be performed due to ataxia and abnormal motor responses, all of which inhibited self-gait. In the ward, high-walker gait training with a caregiver was dangerous due to the patient's falling tendency. We used this robot for gait training in the ward. With the waist strap, the patient demonstrated decreased falling tendency. He could walk with minimal assistance from a caregiver in the ward (Fig. 2C). The private caregiver could independently use the robot without any help from the medical team. At discharge, the patient could walk with a walker under a caregiver's supervision.

\section{DISCUSSION}

To the best of our knowledge, this is the first trial that investigates the effect of using robot in subacute stroke patients in the ward without any assistance from medical team. We found that the use of Robin-T robot allowed patients to have more rehabilitation time. Those who received caregiver driven robot-assisted in-ward training had additional 600 minutes of exercise time. Clinically meaningful improvements in K-MBI, FIM, and FAC scores were observed for most patients. Even in those patients who could not initially stand, assisted standing exercises became feasible. None of these patients reported feeling uncomfortable using these robots. In fact, patients were quite motivated and eager to participate in the exercise program. By using Robin-T robot, patients spent more time exercising which resulted in increased functional recovery. Those patients who were not able to independently receive rehabilitation treatment due to weakness and ataxia after a stroke demonstrated good results from using the robot. They were sufficiently motivated to receive rehabilitation treatment. By eliminating the risk of falls, patients could exercise beyond rehabilitation treatment time in physical therapy room. 
In conventional rehabilitation, a therapist's role is fixed and unmovable. Therefore, most treatment is conducted in a rehabilitation treatment room. For most stroke patients who find it difficult to move, traveling to a facility for rehabilitation is a challenge. Without a main caregiver, it is often impossible for patients to get to their rehabilitation treatment room [10]. Therefore, caregivers and therapists face significant burden. Research is being performed to investigate ways to reduce such burden. A recent study has shown that video-based therapy for postacute stroke patients is safe without negatively impacting independence or being stressful to caregivers [11]. In the same context, the use of Robin-T robot reduced such burden and allowed additional time for rehabilitation treatment in the ward. Thus, structured physical activity programs could be conducted. In addition, previous studies have suggested that caregivers can affect patients' outcomes [12]. Galvin et al. [13] have found that familymediated exercises have positive effects on functional outcome of stroke patients and perceived strain in caregivers. When family-mediated exercises were performed, both patients and caregivers showed physical and psychological benefits from the program [13]. Other studies have suggested that involving caregiver in rehabilitation could reduce the fear about problems that may encounter during caregiving $[7,14]$. A previous trial has been reported that conducting mutual interaction exercise between dementia patient and caregiver could increase pleasure, mood, and self-esteem [15]. These studies suggest that structured patient-caregiver interaction not only affects patients, but also caregivers.

In this study, clinical usage of Robin-T as a sitting device, a standing frame, or a partial body-weight support high-walker was reviewed. Sitting device is a common intervention for individuals who have inadequate trunk control or core instability. It has been proven to be effective in improving dynamic sitting balance [16]. Standing frame is a common intervention for individuals who have inadequate postural control or lower limb strength [17]. It could be used in passive static standing practice. It is frequently applied when independent standing is impossible. Body-weight support systems have been widely used for post-stroke gait rehabilitation [18-20]. These systems can improve body weight distribution between paretic and non-paretic limbs. Therefore, erect posture could be facilitated. They can promote improvement in spatial-temporal gait characteristics, including interlimb symmetry of stance and swing phases, muscle activity, and joint angle excursions of the lower limbs [21]. Gait training may provide a degree of passive movement to the lower limbs with little change in muscular activation, thus limiting the extent of skill transfer to overground walking. Gait training with overground body-weight support has an additional benefit of improving step-length symmetry. In addition, body-weight support training also can promote immediate and durable improvement in paretic step length [22]. Patients' gait ability is considered to be improved based on such aspects. Further research is needed to examine changes in parameters such as gait symmetry, gait velocity, and bilateral coordination.

This study has several limitations. First, the present retrospective case series enrolled a diverse group of stroke patients with weakness and ataxia. Second, locations of lesions in each patient did not coincide. Third, this study did not have a control group. Therefore, it was impossible to discriminate the effect between robot and conventional rehabilitation. Fourth, there was no quantitative survey on the increase time of the exercise and satisfaction of the patient or caregiver. Fifth, only better compliance patients were enrolled. Therefore, the effect of poor compliance patient was unattainable. Although not much evidence can be gleaned from a case series, this is an appropriate method to explain and begin the discussion regarding the ability of this new treatment to improve in-ward stroke rehabilitation. Further research in the form of randomized controlled trials is needed to determine the effect of in-ward robot use to gain insight into which exercise might be the most appropriate for stroke patients and to determine the effect of a combination of different rehabilitation principles and guidelines. Future trials may recruit patients similar to those used in this study using additional outcome parameters such as 6-Minute Walk Tests of gait speed and stride length to make different trials comparable. Additionally, further investigations should examine whether the introduction and combination of other types of therapy in rehabilitation programs could translate into quicker or better recovery for patients after stroke. Large-scale investigations are also needed to confirm our preliminary findings.

It is important to note that it is difficult for some patients to use this robot. Some are too heavy for a caregiver to board them onto the device. Those with severely poor 
sitting balance needed one caregiver to maintain the sitting posture while another caregiver was needed to move the robot. In addition, patients with poor cooperation who hung on the robot were unable to achieve weight bear. Thus, therapeutic effects could not be expected for such patients. Without motivation, treatment with this device is infeasible. Based on our research, the following indications are suggested for this device: (1) patients need sufficient cognitive ability to fully communicate, (2) patients should be motivated for rehabilitation, and (3) patients should be boarded onto the device with the assistance of one caregiver.

In conclusion, utilization of robot device for caregiver driven robot-assisted in-ward training effectively ameliorated stroke patients' ataxia and improved functions of their lower limbs. Caregivers play an important role in the care and continuing rehabilitation of patients after stroke. In subacute stroke patients, the use of robot resulted in improvement in sitting and standing balance as well as gait functions. This study has shown that performing additional rehabilitation treatment with Robin$\mathrm{T}$ robot in the ward is feasible and beneficial for stroke patients. It may offer an approach to improve patient independence and improve patient outcomes.

\section{CONFLICT OF INTEREST}

No potential conflict of interest relevant to this article was reported.

\section{ACKNOWLEDGMENTS}

This study was promoted by the spread business of market creation type robots of the Ministry of Trade, Industry, and Energy. It was carried out using rehabilitation robots supplied by the Ministry of Health \& Welfare and the National Rehabilitation Center in 2014.

\section{REFERENCES}

1. Belagaje SR. Stroke rehabilitation. Continuum (Minneap Minn) 2017;23(1, Cerebrovascular Disease):238-53.

2. Kwakkel G, van Peppen R, Wagenaar RC, Wood Dauphinee S, Richards C, Ashburn A, et al. Effects of augmented exercise therapy time after stroke: a metaanalysis. Stroke 2004;35:2529-39.
3. Teasell RW, Kalra L. What's new in stroke rehabilitation. Stroke 2004;35:383-5.

4. Bhogal SK, Teasell R, Speechley M. Intensity of aphasia therapy, impact on recovery. Stroke 2003;34:98793.

5. Astrand A, Saxin C, Sjoholm A, Skarin M, Linden T, Stoker A, et al. Poststroke physical activity levels no higher in rehabilitation than in the acute hospital. J Stroke Cerebrovasc Dis 2016;25:938-45.

6. Miller EL, Murray L, Richards L, Zorowitz RD, Bakas T, Clark $\mathrm{P}$, et al. Comprehensive overview of nursing and interdisciplinary rehabilitation care of the stroke patient: a scientific statement from the American Heart Association. Stroke 2010;41:2402-48.

7. Vloothuis JD, Mulder M, Veerbeek JM, Konijnenbelt M, Visser-Meily JM, Ket JC, et al. Caregiver-mediated exercises for improving outcomes after stroke. Cochrane Database Syst Rev 2016;12:CD011058.

8. Galvin R, Stokes E, Cusack T. Family-Mediated Exercises (FAME): an exploration of participant's involvement in a novel form of exercise delivery after stroke. Top Stroke Rehabil 2014;21:63-74.

9. Gandolfi M, Geroin C, Tomelleri C, Maddalena I, Kirilova Dimitrova E, Picelli A, et al. Feasibility and safety of early lower limb robot-assisted training in sub-acute stroke patients: a pilot study. Eur J Phys Rehabil Med 2017;53:870-82.

10. Tsunoda T, Maeshima S, Watanabe M, Nagai A, Ueno Y, Ozeki Y, et al. Rehabilitation for a patient with hemiplegia, ataxia, and cognitive dysfunction caused by pontine hemorrhage. Case Rep Neurol 2015;7:213-20.

11. Redzuan NS, Engkasan JP, Mazlan M, Freddy Abdullah SJ. Effectiveness of a video-based therapy program at home after acute stroke: a randomized controlled trial. Arch Phys Med Rehabil 2012;93:2177-83.

12. van den Berg M, Crotty M Prof, Liu E, Killington M, Kwakkel G, van Wegen E. Early supported discharge by caregiver-mediated exercises and e-health support after stroke: a proof-of-concept trial. Stroke 2016;47: 1885-92.

13. Galvin R, Cusack T, O'Grady E, Murphy TB, Stokes E. Family-mediated exercise intervention (FAME): evaluation of a novel form of exercise delivery after stroke. Stroke 2011;42:681-6.

14. Kalra L, Evans A, Perez I, Melbourn A, Patel A, Knapp $\mathrm{M}$, et al. Training carers of stroke patients: randomised 
controlled trial. BMJ 2004;328:1099.

15. Veerbeek JM, van Wegen E, van Peppen R, van der Wees PJ, Hendriks E, Rietberg M, et al. What is the evidence for physical therapy poststroke? A systematic review and meta-analysis. PLoS One 2014;9:e87987.

16. Dean CM, Channon EF, Hall JM. Sitting training early after stroke improves sitting ability and quality and carries over to standing up but not to walking: a randomised trial. Aust J Physiother 2007;53:97-102.

17. Braun T, Marks D, Thiel C, Zietz D, Zutter D, Gruneberg C. Effects of additional, dynamic supported standing practice on functional recovery in patients with sub-acute stroke: a randomized pilot and feasibility trial. Clin Rehabil 2016;30:374-82.

18. Hesse S. Treadmill training with partial body weight support after stroke: a review. NeuroRehabilitation 2008;23:55-65.

19. Combs-Miller SA, Kalpathi Parameswaran A, Colburn
D, Ertel T, Harmeyer A, Tucker L, et al. Body weightsupported treadmill training vs. overground walking training for persons with chronic stroke: a pilot randomized controlled trial. Clin Rehabil 2014;28:873-84.

20. Manning CD, Pomeroy VM. Effectiveness of treadmill retraining on gait of hemiparetic stroke patients. Physiotherapy 2003;89:337-49.

21. Hesse S, Uhlenbrock D, Sarkodie-Gyan T. Gait pattern of severely disabled hemiparetic subjects on a new controlled gait trainer as compared to assisted treadmill walking with partial body weight support. Clin Rehabil 1999;13:401-10.

22. Gama GL, Celestino ML, Barela JA, Forrester L, Whitall J, Barela AM. Effects of gait training with body weight support on a treadmill versus overground in individuals with stroke. Arch Phys Med Rehabil 2017; 98:738-45. 